\title{
THE CONTRIBUTION OF PLEIOTROPY AT THE MOP LOCI TO CONTINUOUS VARIATION IN NICOTIANA RUSTICA
}

\author{
M. K. S. AL-BANNA, J. L. JINKS AND H. S. POONI \\ Department of Genetics, University of Birmingham, PO. Box 363, Birmingham B15 2TT, \\ England \\ Received 18.viii.83
}

\begin{abstract}
SUMMARY
The relationship between the allelic constitution at three major gene loci controlling inflorescence morphology and six continuously varying characters has been investigated in 58 inbred lines derived from the cross of varieties 2 and 12 of Nicotiana rustica by single seed descent. Consistent evidence for a relationship arising from pleiotropy or a linkage disequilibrium is confined to two characters, final plant height $(\mathrm{FH})$ and plant height midway through the growing season (H7). These relationships, which on average account for only 23 and 15 per cent of the additive genetic variation in $\mathrm{FH}$ and $\mathrm{H} 7$, respectively, are attributable to the phenotypic difference between two alternative forms of the inflorescence, mop and non mop, rather than to a direct effect of gene dosage at the three controlling loci. Since the mop:non mop difference is controlled by allelic differences at the three loci that display triplicate interactions, the variation in $\mathrm{FH}$ and $\mathrm{H} 7$ associated with it should display interactions of the same kind. But, since the allelic differences at the three loci are in the association phase in varieties 2 and 12, they cannot account for the majority of the non-allelic interactions for $\mathrm{FH}$ and $\mathrm{H} 7$ in this cross, as these are controlled by allelic differences in the dispersion phase.
\end{abstract}

\section{INTRODUCTION}

Fifty-eight of a random sample of 60 highly inbred lines derived from the cross of varieties 2 and 12 of Nicotiana rustica (Jinks, Jayasekara and Boughey, 1977) have been classified on the basis of the three locus model of the genetical control of the mop:non mop difference in inflorescence morphology proposed by Jinks and Coombs (1979). The 58 lines fall into four groups of 4, 18, 30 and 6 lines on the basis of homozygosity for the recessive allele $(\mathrm{mm})$ at $0,1,2$ and all three loci, respectively. We can, therefore, examine whether the dosage of dominant to recessive alleles at these loci contribute to the considerable continuous variation displayed by the inbred lines derived from the cross of varieties 2 and 12 (Pooni, Jinks and Jayasekara, 1978). Any variation attributable to the pleiotropic action of these major genes is amenable to classical gene action analysis, the results of which can be compared with biometrical genetical analyses of the total variation.

\section{MATERIAL AND METHOD}

Fifteen plants of each of the 60 inbred lines (referred to as the D lines) were raised in a completely randomised design in 1976, 1977 and 1978 and with one line omitted in 1979. In all four seasons four characters, height at four weeks (H2) final plant height (FH), maximum leaf length $(\mathrm{LL})$ and 
flowering time (FT) were recorded. In addition, in 1976, 1977 and 1979 total seed capsule number per plant (TCN) was recorded and in 1978 and 1979 plant height in the middle of the season (H7) was also recorded.

For each character in each season, the total sum of squares for the 58 of the $60 \mathrm{D}$ lines which had been classified in respect of the allelic differences at the mop loci (57 in 1979) was partitioned into sums of squares for differences between lines for 57 degrees of freedom (56 in 1979) and for differences between replicate plants within lines for $58 \times 14$ degrees of freedom $(57 \times 14$ in 1979). The sum of squares between lines was further partitioned into that between the four groups (homozygous for mop allele at $0,1,2$ and 3 loci) for three degrees of freedom and that between lines within the four groups for $3+17+29+5=54$ degrees of freedom (53 in 1979).

If the mean square between the 58 (or 57) lines is significant when tested against that within the lines there is additive genetic variation for the character and its magnitude equals the components of variance between lines $\left(\sigma_{l}^{2}\right)$. If the mean square between the four groups is significant when tested against that between lines within groups a significant portion of the additive genetic variation is associated either through pleiotropy or a linkage disequilibrium with the allelic differences at the mop loci. The magnitude of this portion is the reduction in the additive genetic variation when it is reestimated from the component of variance between lines within groups $\left(\sigma_{l g}^{2}\right)$.

To test whether any significant association is related to the phenotype of the inflorescence or to the dosage of the mop allele, the sum of squares between the four groups for three degrees of freedom can be further partitioned in two ways:

The first partitioning based on the mop and non mop phenotypes divides the four groups into two, one consisting of the group which is homozygous for the recessive mop allele at all three loci and the other consisting of the remaining three groups which are homozygous for this allele at two or fewer of the three loci. This partitioning leads to a sum of squares for the difference between the mop and non mop lines for one degree of freedom with the remainder sum of squares for two degrees of freedom for the difference between the three groups which have the non mop phenotype (table 1).

The second partitioning is achieved by a regression analysis in which the four group means are regressed onto the number of loci which are homozygous for the mop allele $(0,1,2$ and 3$)$. With four groups there is one degree of freedom for each of the linear, quadratic and cubic components of the regression.

Previous analyses (Jinks and Coombs, 1979) have suggested that the phenotypic difference between the mop and non mop inflorescences has a more marked effect on the developmental relationship between flowering time and final height than on the variation for either character alone. The correlation between FT and FH has therefore been estimated for the inbred lines within each of the four groups and within the mop and non mop phenotypic classes (table 2). 


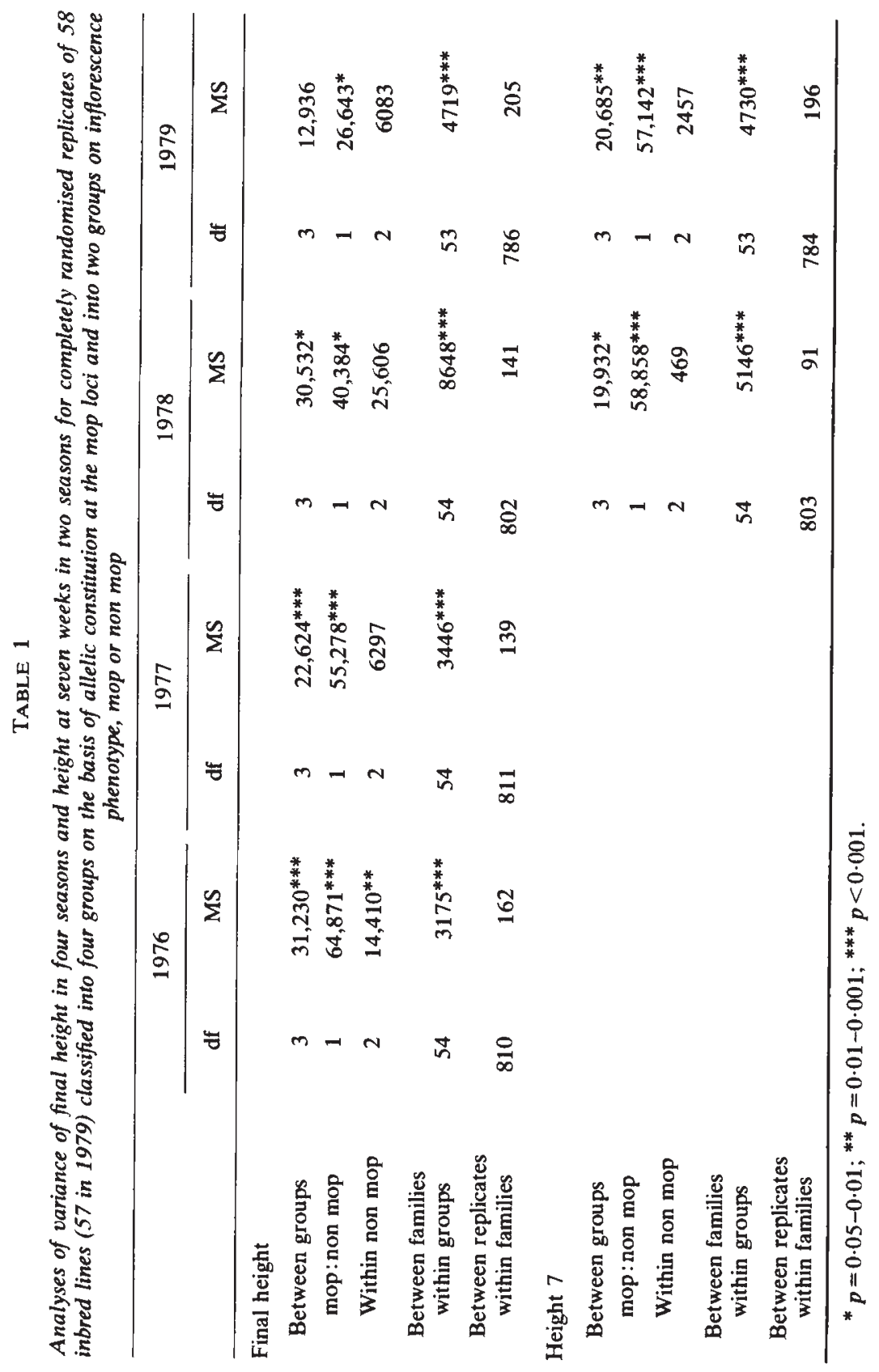




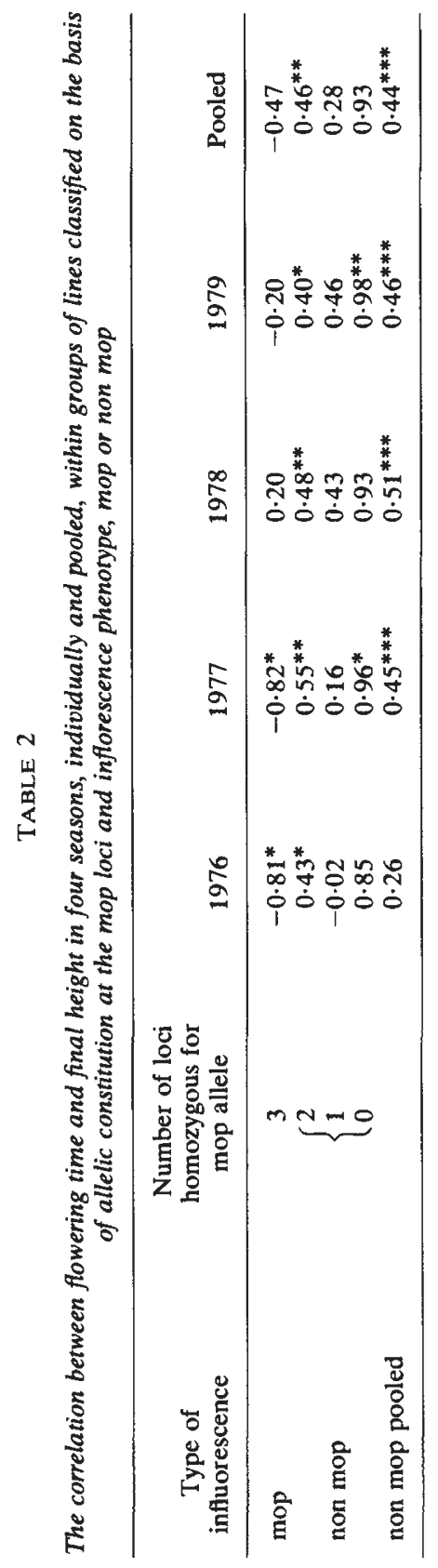




\section{RESULTS}

The analyses of variance showed highly significant differences between the inbred family means for all characters in all of the seasons (except $\mathrm{H} 2$ in 1976) in which they were recorded (see section 2). There is, therefore, considerable additive genetic variation among the inbred lines as previously reported (Pooni, Jinks and Jayasekara, 1978). With the exception of one season (1976), however, there are no significant differences between the four groups of inbred lines for H2, LL, FT, and TCN. In complete contrast, the difference between the four groups is significant in every season for $\mathrm{FH}$ (except in 1979) and H6 (table 1). Further analysis will, therefore, be confined to these two characters.

When the sum of squares between the four groups is partitioned by the first method (section 2), the mean square between mop and non mop is significant for both $\mathrm{FH}$ and $\mathrm{H} 7$ in every season. The mean square between the three groups within the non mop class is, however, non significant with the exception of $\mathrm{FH}$ in one season (table 1). The alternative partitioning by regression analysis gave significant linear and quadratic components for the regression onto allele dosage at the mop loci in three of the four seasons for $\mathrm{FH}$ and in both seasons for $\mathrm{H} 7$. No cubic component was significant.

Estimates of the additive genetic variance $D$ (or more strictly $D+I$ in the presence of epistasis-see section 4 ) and of the additive genetic variance associated with the mop loci $D_{\text {mop }}$ are listed in table 3.

TABLE 3

Estimates of the additive genetic variance $D($ or $D+I)$ and of the portion of it, $D_{\text {mop }}$ associated with the mop loci for final height in four seasons individually and pooled and for height at seven weeks in two seasons

\begin{tabular}{cccccc}
\hline & \multicolumn{2}{c}{ Final height } & & \multicolumn{2}{c}{ Height 7} \\
\cline { 2 - 3 } \cline { 5 - 6 } Season & $D$ & $D_{\text {mop }}$ & & $D$ & $D_{\text {mop }}$ \\
\hline 1976 & 299.98 & 98.67 & & \\
1977 & 288.06 & 67.38 & & \\
1978 & 651.40 & 78.15 & & 392.91 & 52.43 \\
1979 & 335.04 & 29.76 & & 365.29 & 57.93 \\
Pooled & 313.91 & 74.24 & & \\
\hline
\end{tabular}

In spite of seasonal variation, the correlations between FT and FH (table 2) show a tendency towards negative correlations among lines with the mop phenotype and positive correlations among lines with the non mop phenotype as previously reported (Jinks and Coombs, 1979). This contrast is particularly pronounced between the two extreme genotypic groups in respect of the mop loci ( 3 and 0 in table 2). But while the two intermediate genotypic groups ( 2 and 1 ) have intermediate correlations, there is no direct relationship over all four groups between the correlation and allele dosage at the mop loci. A possible explanation is the genetical heterogeneity within the two intermediate groups, each of which contains three different genotypes in respect of the mop loci. 


\section{INTERPRETATION AND DISCUSSION}

For only two of the six characters studied, namely $\mathrm{FH}$ and $\mathrm{H7}$, is there consistent evidence of an influence of the allelic differences at the three mop loci on their additive genetic variation. The analyses of variance (table 1) and the four group means averaged over seasons (table 4) show that this influence is strongly associated with the phenotypic difference between the mop and non mop types of inflorescence. There is little significant evidence (table 1, FH 1976) of any effect of the differences between the three genotypes that lead to the non mop phenotype, although the mean of the group which is homozygous for the mop allele at only one of the three loci ( 1 in table 4 ) is higher than that of the other two non mop groups for both FH and $\mathrm{H} 7$ ( 2 and 0 in table 4 ). In these circumstances, the non linearity of the regression of $\mathrm{FH}$ and $\mathrm{H} 7$ on the dosage of the mop allele is no more than is expected.

TABLE 4

The mean final height and height at seven weeks of inbred lines grouped on the basis of the mop and non mop types of inflorescence and of the genotypic constitution in respect of the three mop loci

\begin{tabular}{|c|c|c|c|}
\hline $\begin{array}{c}\text { Type of } \\
\text { influorescence }\end{array}$ & $\begin{array}{l}\text { Number of loci } \\
\text { homozyous for } \\
\text { mop allele }\end{array}$ & Final height & Height 7 \\
\hline mop & 3 & $90 \cdot 19$ & 53.79 \\
\hline non mop & $\left\{\begin{array}{l}2 \\
1 \\
0\end{array}\right.$ & $\begin{array}{l}110 \cdot 47 \\
121 \cdot 91 \\
110 \cdot 46\end{array}$ & $\begin{array}{l}81 \cdot 89 \\
83 \cdot 49 \\
76 \cdot 53\end{array}$ \\
\hline mean non mop & & $114 \cdot 28$ & $80 \cdot 64$ \\
\hline linear regression coefficient & & $-7 \cdot 22$ & $-6 \cdot 99$ \\
\hline quadratic regression coefficient & & $-7 \cdot 93$ & $-8 \cdot 76$ \\
\hline
\end{tabular}

The estimates of the portion of the additive genetic variation associated with the mop loci $\left(D_{\text {mop }}\right)$ vary from 9 to 33 per cent for $\mathrm{FH}$, with an overall value of 23 per cent from an analysis of the data pooled over seasons and from 13 to 16 percent for $\mathrm{H} 7$ (table 3 ). Thus, even if all of the variation associated with the mop loci is ascribed to pleiotropy rather than to a linkage disequilibrium, the allelic differences at these loci account for only a small part of the variation in $\mathrm{FH}$ and $\mathrm{H7}$ and none that is consistently detectable for the other four characters.

The part of the variation in $\mathrm{FH}$ and $\mathrm{H7}$ which may be pleiotropic is largely attributable to the phenotypic difference between the mop and non mop inflorescence (table 1). Since this phenotypic difference is controlled by allelic differences at the mop loci that display classical duplicate (or triplicate) epistasis (Jinks and Coombs, 1979) the pleiotropic variation in $\mathrm{FH}$ and $\mathrm{H} 7$ must also display this form of non-allelic interaction. Put another way, that part of the variation in $\mathrm{FH}$ and $\mathrm{H} 7$ that may be pleiotropic shows a non linear relationship with gene dosage at the mop loci such that there is little change in $\mathrm{FH}$ and $\mathrm{H} 7$ on substituting the mop allele for the non mop allele until all three loci are simultaneously homozygous for the mop allele. A non linear relationship of this kind is the biometrical genetical 
generalisation to more than two loci of classical duplicate interactions (Mather and Jinks, 1971, 1982).

All analyses of the genetical variation for $\mathrm{FH}$ and $\mathrm{H} 7$ in the cross of varieties 2 and 12 since the first analysis of Jinks (1956) have detected non-allelic interaction of an apparently duplicate kind (see Jinks, 1983 for review). It is possible, therefore, that this non-allelic interaction is no more than the pleiotropic effect of the duplicate interactions at the mop loci. The recent analyses of this cross by Pooni and Jinks (1981 and 1983), however, make this extremely unlikely. Thus the allelic differences at the mop loci are in the association phase in varieties 2 and 12, the former being homozygous for all of the mop alleles and the latter for all of the non mop alleles. Pooni and Jinks have shown that much of the non-allelic interaction for $\mathrm{FH}$ and $\mathrm{H7}$ in this cross arises from allelic differences which are in the dispersed phase in these varieties.

As previously reported by Jinks and Coombs (1979) the effect of the allelic differences at the mop loci on the developmental relationships between FT and FH can be more profound than their effect on the two characters individually. Thus we have been able to confirm the reversal of the relationship between FT and FH in inbred lines which are homozygous mop at all loci and those which are homozygous non mop at these loci. Thus among the lines which are homozygous mop at all loci, those which flower earlier are the taller at the end of the season while among the lines which are homozygous non mop at all loci, those which flower earlier are shorter.

The inbred lines on which our analyses are based were derived by single seed descent from the $F_{2}$. There have, therefore, been repeated opportunities for recombination during their derivation. They are not, however, expected to be in linkage equilibrium for tightly linked genes (Jinks and Pooni, 1982). We must not forget, therefore, that the variation in $\mathrm{FH}$ and $\mathrm{H} 7$ which we have examined in terms of pleiotropy may include contributions of genes in linkage disequilibrium with the allelic differences at the mop loci.

The cross of varieties 2 and 12 is the most heterotic, segregates to give the greatest amount of genetical variation and displays the most complex gene action of any that have been examined in Nicotiana rustica. It is likely, therefore, that in this cross we are observing the segregation of allelic differences at many if not at most of the loci that regularly contribute to the continuous variation in $N$. rustica. Equally it is likely that in this cross we are observing the contributions of allelic differencs at these loci that are as large as or larger than those in other crosses. In attempting to understand the hereditary determinants of continuous variation it is significant, therefore, that in the cross of varieties 2 and 12 the only major genes known to contribute to the quantitative variation, those at the mop loci, contribute, at most, only a small part of the variation in two characters and none in another four. Pleitropic effects of major genes are not an important contribution.

\section{REFERENCES}

JINKS, J. L. 1956. The $\mathrm{F}_{2}$ and backcross generations from a set of diallel crosses. Heredity, 10, $1-30$. 
JINKS, J. L. 1983. Biometrical genetics of heterosis. Monographs on Theoretical and Applied Genetics, Vol. 6. Heterosis ed. R. Frankel. Springer-Verlag, Berlin.

JINKS, J. L., JA YASEKARA, N. E. M. AND BOUGHEY, H. 1977. Joint selection for both extremes of mean performance and sensitivity to a macroenvironmental variable. II. Single seed descent. Heredity, 39, 345-355.

JINKS, J. L. AND COOMBS, D. 1979. The relationship between major gene controlled inflorescence morphology and continuous variation for the final height in Nicotiana rustica. Heredity, 42, 229-307.

JINKS, J. L. AND POONI, H. S. 1982. Predicting the properties of pure breeding lines extractable from a cross in the presence of linkage. Heredity, 49, 265-270.

MATHER, K. AND JINKS, J. L. 1971. Biometrical Genetics. 2nd edn. Chapman and Hall, London.

MATHER, K. AND JINKS, J. L. 1982. Biometrical Genetics. 3rd edn. Chapman and Hall, London.

POONI, H. S., JINKS, J. L. AND JAYASEKARA. N. E. M. 1978. An investigation of gene action and genotype $\times$ environment interaction in two crosses of Nicotiana rustica by triple test cross and inbred line analysis. Heredity, 41, 83-92.

POONI, H. S. AND JINKS, J. L. 1981. The true nature of the non-allelic interactions in Nicotiana rustica revealed by association crosses. Heredity, 47, 253-258.

POONI, H. S. AND JINKS, J. L. 1983. Comparison of associated and dispersed testers in the triple test cross. Heredity, 50, 73-83. 